\title{
RBF-Network-Based Sliding Mode Control
}

\author{
Sinn-Cheng Lin ${ }^{1}$, Student Member IEEE and Yung-Yaw Chen ${ }^{1,2}$, Member IEEE \\ ${ }^{1}$ Department of Electrical Engineering, Lab. 202, National Taiwan University, \\ Taipei, Taiwan, R.O.C. \\ ${ }^{2}$ Electronic Research Laboratory, University of California, Berkeley, \\ CA 94720, USA
}

\begin{abstract}
A sliding mode controller (SMC) design method based on radial basis function network (RBFN) is proposed in this paper. Similar to the multilayer perceptron, the RBFN also known to be good universal approximator. In this work, the weights of the RBFN are changed according to some adaptive algorithms for the purpose of controlling the system state to hit an user-defined sliding surface and then slide along it. The initial weights of the RBFN can be set to small random numbers, and then on-line tuned automatically, no supervised learning procedures are needed. By applying the RBFN-based sliding mode controller to control a nonlinear unstable inverted pendulum system, the simulation results show the expected approximation sliding property was occurred, and the dynamic behavior of control system can be determined by the sliding surface.
\end{abstract}

\section{Introduction}

Sliding mode control (SMC) strategy has been developed essentially in the literature from the Soviet Union [3][4]. By carefully selecting a suitable sliding surface and applying discontinuous control action across the surface, the resulting state trajectory of the control system can be transferred onto the sliding surface and then slide along the surface. There are two phases in the sliding mode control system: 1) hitting phase and 2) sliding phase. In the hit- ting phase, a hitting control law will be applied to drive the representation point everywhere of state space onto the sliding surface. As soon as the representation point hit the surface, the controller turns the sliding phase on, and applies an equivalent control law to keep the state on the sliding surface. In ideal situation, when the state trajectory stay on the sliding surface, the control system then has perfect insensitivity respect to the external disturbances as well as the plant parameter variations. Furthermore, even if the system is perturbed such that the representation point is out off the sliding surface, the control system turns on the hitting phase again, and reacts the hitting controller to bring the state toward the sliding surface once more. Therefore, the sliding mode control imposes certain constrains on system dynamics. In other words, one can predetermine the sliding surface which dominates the dynamic behavior of control system. However, without knowing the mathematical model of system, there is no way to obtain the equivalent control. In the real world, there are many complex industrial processes whose available models can't be easily developed. Hence, it's a tough job that designing a modelbased sliding mode controller to deal with illdefined systems. In this paper, we develop a new sliding mode control design strategy based on radial basis function network (RBFN) [1][2]. The weights of the RBFN are changed according to some adaptive algorithm for the purpose of controlling the system states 
to hit an user-defined sliding surface and then slide along it. The initial weights of the RBFN can be set to small random numbers, and then on-line tuned, no supervised learning procedures are needed.

This paper is organized as follows. Section II is a brief description of sliding mode control. In Section III, an adaptive radial basis function networks is presented for sliding mode controller design. In Section IV, the proposed controller is applied to a nonlinear and unstable inverted pendulum system, the simulation results verify the efficiency of proposed approach. Conclusions are given in Section V.

\section{The Sliding Mode Control}

Consider a class of $n$-th order nonlinear systems, whose dynamics can be represented in the following differential equation

$$
y^{(n)}=f\left(y, \dot{y}, \ldots, y^{(n-1)}\right)+b u, \quad b>0
$$

where $f($.) is a unknown continuous function, $b$ is a unknown positive constant, and $u \in R$ is the system input and $y \in R$ is the system output. To transfer this differential equation to a state space realization, let $r$ be the reference input, and let $\varepsilon=r-y$ be the error signal. Define the state $e_{i}=r^{(i-1)}-y^{(i-1)} \triangleq \varepsilon^{(i-1)}$, $i=1,2, \ldots, n$, then the system (1) can be represented as the following state equations

$$
\left\{\begin{array}{l}
\dot{e}_{i}=e_{i+1}, \\
\dot{e}_{n}=r^{(n)}-f-b u
\end{array} \quad i=1, \ldots, n-1\right.
$$

In conventional SMC design, the designer must choose a sliding function. In this paper, we adopt the linear sliding function $s=\mathbf{c}^{\mathrm{T}} \mathbf{e}$, where $\mathbf{c}=\left[\begin{array}{lll}c_{1} & c_{2} & \ldots, \\ c_{n-1} & 1\end{array}\right]^{\mathrm{T}}$. While the state reaches the sliding surface $s=0$, the equivalent control, $u_{e q}$, could keep the state stay on the sliding surface. So, $u_{e q}$ could be derived from setting the derivative of $s, \dot{s}$, equal to zero, that is

$$
u_{e q}=\left.u\right|_{s=0}=b^{-1}\left(-f+r^{(n)}+\overline{\mathbf{c}}^{\mathrm{T}} \mathbf{e}\right)
$$

where $\overline{\mathbf{c}}=\left[\begin{array}{lllll}c_{1} & c_{2} & \ldots & c_{n-1} & 0\end{array}\right]^{\mathrm{T}}$. Moreover, in the sliding mode, $s=0$, the system dynamic could be characterized by the following characteristic polynomial

$$
D^{n-1}+c_{n-1} D^{n-2}+\ldots+c_{1}=0
$$

where $D \stackrel{\Delta}{=} \frac{d}{d t}$. Therefore, with suitable choice of the coefficients $c_{i}$, one can obtain a desired equivalent control system .

\section{Adaptive Radial Basis Function Networks} for Sliding Mode Controller Design

Figure 1 shows the block diagrams of RBF network [1], the basis functions we adopted in this paper are all in the form of $g(\mathbf{x})=\exp \left[-\left(\frac{\mathbf{x}-m}{\sigma}\right)^{2}\right]$. Sometime, for the convenience of notational simplicity, function $g($.) also expressed as $G(m, \sigma)$ in this paper. The output of the network is

$$
u=\sum_{i=1}^{N} w_{i} g_{i}(\mathbf{x}) \stackrel{\Delta}{=} \mathbf{w}^{\mathrm{T}} \mathbf{g}(\mathbf{x})
$$

where $\mathbf{w}=\left[\begin{array}{llll}w_{1} & w_{2} & \ldots & w_{N}\end{array}\right]^{\mathrm{T}}, \mathbf{g}=\left[g_{1} g_{2} \ldots g_{N}\right]^{\mathrm{T}}$.

Without knowing $f($.$) and b$, there is no way to get $u_{e q}$, the main purpose here is to use an adaptive RBF network to approximate it. Suppose that the control law has two parts

$$
u=u_{r}+u_{h}
$$

where $u_{r}$ is obtained from a RBF network described in the last section. Now, we derived the weights updating law to adjust the weight vector $\mathbf{w}$. Assume there exist a set of weights $\mathbf{w}^{*}$ in the universe $W$, such that $\left(\mathbf{w}^{*}\right)^{\mathrm{T}} \mathbf{g}-u_{e q}$ is minimal. Define a Lyapunov function

$$
V=\frac{1}{2} s^{2}+\frac{b}{2 \eta} \phi^{\mathrm{T}} \phi
$$


where $\phi \stackrel{\Delta}{=} \mathbf{w}^{*}-\mathbf{w}$, and $\eta$ is a positive constant. If we choose the weights updating law as

$$
\dot{\mathbf{w}}=\eta s(\mathbf{e}) \mathbf{g}(s)
$$

and the sign of $u_{h}$ is agree with that of $s b$ (this part will be derived in the next paragraph), then $\dot{V}$ will has a negative upper bound.

Stability consideration: The purpose of the hitting control part, $u_{h}$, in the equation (6) is used to draw the state to hit the sliding surface no matter where the initial state is. To achieve such a goal, let's define a Lyapunov function for $s$

$$
V_{s}=\frac{1}{2} s^{2}
$$

the hitting condition which guarantees the stability of sliding mode control system is $\dot{V}_{s}=s \dot{s}<-\eta|s|$ [7]. Suppose that we know the upper bound of $f($.) and lower bound of $b$, i.e. $|f| \leq \bar{f}$, and $0<\underline{b} \leq b$, then

$u_{h}=-\operatorname{sign}(s)\left[\underline{b}^{-1}\left(\bar{f}+\left|r^{(n)}\right|+\left|\overline{\mathbf{c}}^{\mathrm{T}} \mathbf{e}\right|+\eta\right)+\left|u_{r}\right|\right]$

yields that $\dot{V}_{s} \leq 0$, and the hitting condition could be satisfied, therefore the system is stable.

Practical consideration: In general, the hitting control part described previously is a high gain bang-bang control. It will generate a very large control force and increase the control cost, which is usually undesired. Therefore, we replace the sign function in (9) by a saturation function and modify the control law (6) by

$$
u=u_{r}+\alpha u_{h}
$$

where $\alpha=\left\{\begin{array}{ll}1, & |s|>\bar{s} \\ 0, & |s|<\bar{s}\end{array}\right.$, and $\bar{s}$ is a constant specified by the designer. Another alternative way to get a better hitting control law by a fuzzy rule base had been developed in our previous work [4].

\section{Simulation Results}

In this section, we applied the RBFN-based SMC developed in the last section to control an inverted pendulum system (Figure 2). The dynamics of the inverted pendulum system could be characterized by two state variables: $x_{1}$ denotes the angle of the pole with respect to the vertical line, and $x_{2}$ denotes the angular velocity of the pole. Neglecting the coefficient of friction, the state equation, which was used for computer simulation, could be expressed as follows

$$
\begin{aligned}
& \dot{x}_{1}=x_{2} \\
& \dot{x}_{2}=\frac{g \sin x_{1}-\cos x_{1}\left(\frac{m l}{m_{c}+m l} x_{2}^{2} \sin x_{1}-\frac{1}{m_{c}+m} f\right)}{\frac{4}{3} l-\frac{m l}{m_{c}+m} \cos ^{2} x_{1}}
\end{aligned}
$$

where $g$ is the gravity constant, $9.8 \mathrm{~m} / \mathrm{s}^{2}, m_{c}$ is the mass of the cart, $1.0 \mathrm{~kg}, m$ is the mass of the pole, $0.1 \mathrm{~kg}, l$ is the length of the pole, $1 \mathrm{~m}$ and $f$ is the input force to the cart. Select $\mathbf{c}=[2$ $1]^{\mathrm{T}}$ and $\eta=100$. The activation functions of the single input node and the single output node are all identical. The number of the hidden nodes is equal to 5 , and their activation functions (the radial basis functions) are selected as $G_{l}(2,1), G_{2}(0.5,0.5), G_{3}(0,0.5)$, $G_{4}(-0.5,0.5)$, and $G_{5}(-2,1)$, respectively. The initial weights are all set to zero. By applying the weights updating law (8) and the control law (10) with $\alpha=0$, i.e. without hitting control, Figure 3 shows the response of the control system. By adding the hitting control part to the system, Figure 4 shows an asymptotically sliding mode can be achieved. 


\section{Conclusions}

In this report, we developed an RBFN-based sliding mode controller, which has the following characteristics

1) Does not require the mathematical model of the system;

2) By applying the hitting control part, the stability of control system can be guaranteed;

3) Approximation sliding mode was occurred;

4) Dynamic behavior of the control system can be specified by an user-defined sliding surface;

5) Does not need any supervise learning procedure. Real time control requirement could be achieved.

Our future research topics focus on hardware implementation of proposed approach and applied to MIMO systems.

\section{Reference}

[1] S. Chen, C. F. N. Cowan, and P. M. Grant, "Orthogonal Least Squares Learning Algorithm for Radial Basis Function Networks," IEEE Trans. on Neural Networks, vol. 2, no. 2, 1991, pp. 302-309.

[2] J. S. Roger and C. T. Sun, "Functional equivalence between radial basis function networks and fuzzy inference systems", IEEE Tran. on Neural Net., vol.4, no.1, pp. 156- 159, JAN, 1993.

[3] V. I. Utkin, Sliding modes and their application in variable structure system, Moscow: Mir, 1978 (English translation)

[4] U. Itkis, Control system of variable structure. New York: Wiley, 1976.

[5] S. C. Lin and C. C. Kung, "A linguistic fuzzy sliding mode controller," Proc. of 1992 A.C.C., pp. 1904-1905.

[6] S. C. Lin and Y. Y. Chen, "Design of adaptive fuzzy sliding mode for nonlinear system control," 3rd IEEE International
Conference on Fuzzy Systems, Orlando, 1994. (to be appered)

[7] J. J. E. Slotine and W. Li, Applied Nonlinear Control, Englewood Cliffs, NJ: Prentice Hall, 1991.

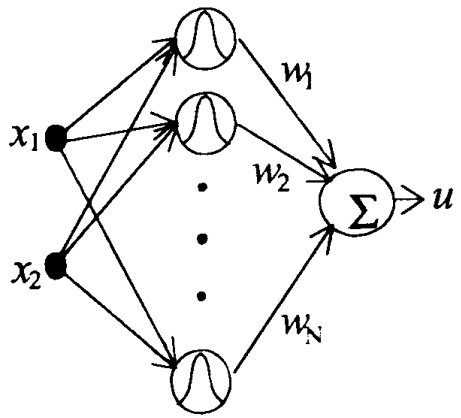

Figure 1 The RBF Network

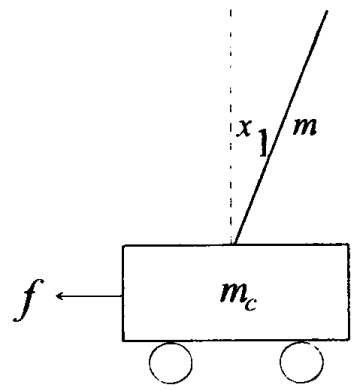

Figure 2 The inverted pendulum system

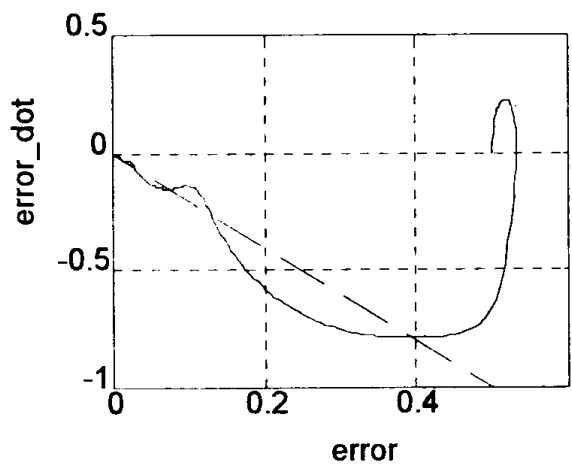

Figure 3 State trajectory of controlled system with $\alpha=0$ 


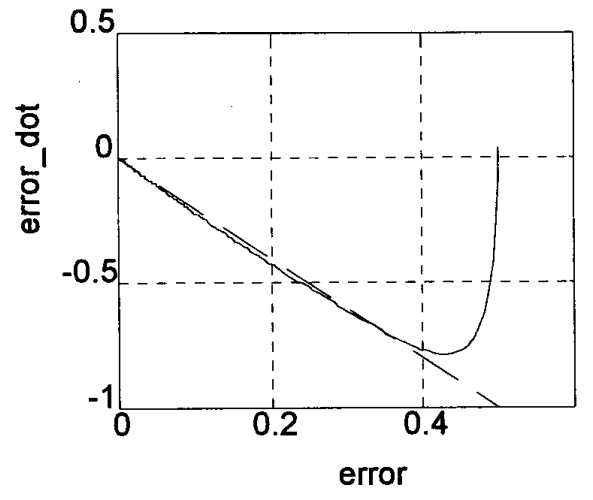

Figure 4 State trajectory of controlled system with $\alpha=1$ 